\title{
Factors Influencing Warm Ischemia Time in Robot- assisted Partial Nephrectomy Change Depending on the Surgeon's Experience
}

Kazuyuki Numakura ( $\sim$ nqf38647@nifty.com )

Akita University Graduate School of Medicine

Mizuki Kobayashi

Akita University Graduate School of Medicine

Atsushi Koizumi

Akita University Graduate School of Medicine

Soki Kashima

Akita University Graduate School of Medicine

Ryohei Yamamoto

Akita University Graduate School of Medicine

Taketoshi Nara

Akita University Graduate School of Medicine

Mitsuru Saito

Akita University Graduate School of Medicine

Shintaro Narita

Akita University Graduate School of Medicine

Takamitsu Inoue

International University of Health and Welfare, Narita Hospital

Tomonori Habuchi

Akita University Graduate School of Medicine

\section{Research Article}

Keywords: RENAL Score, Kidney, Oncology, Robotic surgery

Posted Date: September 1st, 2021

DOI: https://doi.org/10.21203/rs.3.rs-849289/v1

License: (1) (1) This work is licensed under a Creative Commons Attribution 4.0 International License.

Read Full License 


\section{Abstract}

INTRODUCTION: Warm ischemia time (WIT) is a primary concern for robot-assisted laparoscopic partial nephrectomy (RALPN) patients because longer WIT is significantly associated with postoperative deteriorating kidney function. Tumor complexity, determined by the RENAL nephrometry score (RENAL score), can help predict surgical outcomes, but it is unclear what RENAL score and clinical factors affect WIT. This study explored the clinical factors predicting long WIT in RALPN.

MATERIALS AND METHODS: In our institute, 174 RALPNs were performed between November 2013 and February 2021, of which 114 were performed by a single surgeon and included in this study. Clinical staging and the total RENAL score were determined based on preoperative CT scans. The cases were divided into three groups based on experience: period 1: 1-38, period 2: 39-76, and period 3: 77-114. The clinical factors associated with longer WIT were analyzed per period.

RESULTS: The overall median tumor diameter was $32 \mathrm{~mm}$, and one patient had a positive surgical margin, but there were no cancer-related deaths. In total, there were 18 complications (15.8\%). Periods 2 and 3 had larger tumor diameters $(p<0.01)$ and worse preoperative kidney function $(p=0.029)$ than period 1. A RENAL L-component score of 3 was associated with longer WIT in period 3 (odds ratio: 3.900; $95 \%$ confidence interval: $1.004-15.276 ; p=0.044)$, but the tumor diameter and the total RENAL score were not.

CONCLUSIONS: A large tumor in the central lesion indicated by the RENAL L-component score was associated with increased WIT in RALPN.

\section{Introduction}

After the introduction of the robotic platform for kidney surgery, robot-assisted laparoscopic partial nephrectomy (RALPN) for T1 renal cell carcinoma (RCC) became a standard procedure [1, 2]. There are obvious advantages to robotic surgery over pure laparoscopic nephrectomies, such as more precise resections and proper parenchyma suturing [3]. The robotic procedure also achieves good oncological outcomes, preserves more kidney function, and avoids complications [4].

A trifecta of RALPN was defined as warm ischemia time (WIT) $\leq 25$ min, negative surgical margins, and no postoperative complications $\geq$ grade 2 , This is a useful evaluation method for assuring good clinical outcomes in RALPN patients [5]. Of the three factors, WIT should be the most concerning because positive surgical margins and severe complications are quite rare. Moreover, a longer WIT is a major factor associated with deteriorating kidney function [6], and potentially leading to worse survival rates due to chronic kidney disease and cardiovascular disease [7].

The RENAL nephrometry score (Radius [tumor size], Exophytic/endophytic properties, Nearness of the tumor to the collecting system, Anterior/posterior, Location relative to the polar line; RENAL score) is used to predict surgical difficulty in RALPN and laparoscopic partial nephrectomy patients [8]. However, it 
remains unclear what RENAL score or other clinical factors affect WIT in RALPN $[9,10]$. Since most previous studies were assessed a predicting factor of WIT only depending on dividing patients into groups by clinical factors and/or surgical techniques, a real factor could be covered up due to many confounders [11-13]. To minimize this influence, we assessed WIT by dividing patients into three chronological periods, which could reflect a learning curve [14], in the same surgical procedure by a single surgeon. This study aimed to delineate clinical factors predicting longer WIT during RALPN.

\section{Materials And Methods}

Between November 2013 and February 2021, six surgeons performed 174 RALPNs for clinical T1 kidney tumors in our institute. Of these, 114 RALPNs were performed by a single surgeon (T.H.) and were analyzed in this study. The clinical staging and the RENAL score were determined based on CT scans.

RALPN was performed using standard transperitoneal or retroperitoneal approaches depending on the patient's history of trans-abdominal surgery, the tumor size, and the tumor location. Partial nephrectomy was performed by clamping the main trunk of a renal artery(s) with a bulldog clamp. In renal hilar tumors, a renal vein was also clamped. Tumors with a 5 -mm surgical margin were resected under $15 \mathrm{~mm} \mathrm{Hg} \mathrm{CO}$ pressure, while the preparation, renal capsule, and tumor surface were exposed under $10 \mathrm{~mm} \mathrm{Hg} \mathrm{CO} 2$ pressure. Both the inner and outer-layer renorrhaphy were performed by $2-0$ STRATAFIX ${ }^{\mathrm{TM}}$ Spiral PDS ${ }^{\mathrm{Tm}}$ Plus (Ethicon, USA) or $3-0$ V-Loc ${ }^{\text {TM }}$ (Medtronic, Ireland), according to the tumor size and location. All specimens were removed using an ENDOPOUCH ${ }^{\text {TM }}$ RETRIEVER $^{\text {TM }}$ Specimen bag (AutoSuture, UK) through a camera port. Intra-and postoperative complications were categorized based on the Satava [15] and the Clavien-Dindo [16] classification systems, respectively, into major or minor complications; major complications were defined as grade II or higher. To assess clinical risk factors for prolonged WIT, the patients were divided into three groups based on the number of RALPNs in chronological order (period 1: cases 1-38, period 2: cases 39-76, and period 3: cases 77-114).

All procedures were performed according to the tenets of the 1964 Declaration of Helsinki. Informed consent was received for all the participating patients. Research involving human participants was supervised and approved by the Ethics Board of the Akita University Hospital.

All data were expressed as the median (range). The clinical risk factors associated with a longer WIT were screened by univariate analysis. The factors with a $p$-value of $<0.1$ in the univariate analysis were confirmed by logistic regression analysis. All $p$-values were two-sided, and $p<0.05$ was considered statistically significant. All statistical analyses were performed using SPSS version 26.0 statistical software (SPSS Japan Inc., Tokyo, Japan).

\section{Results}

Tables 1 and 2 present the clinicopathological characteristics of all enrolled patients. The tumor diameter increased with each period (median; $25.7 \mathrm{~mm}$ vs. $33.9 \mathrm{~mm}$ vs. $38.9 \mathrm{~mm}, \mathrm{p}<0.01$ ), but there was no 
difference in WIT (17.2 $\mathrm{min}$ vs. $18.4 \mathrm{~min}$ vs. $17.4 \mathrm{~min}, \mathrm{p}=0.666$ ). This is perhaps because the indications for tumor diameter and kidney function extended with increasing surgical experience. In period 1, only one patient $(2.6 \%)$ had a tumor stage higher than $\mathrm{lb}$, but the incidence rate increased in period 2 (8 patients, $21.1 \%)$ and period 3 (18 patients, $47.4 \%$; $(p<0.01$ [chi-squared test]). There was no difference in the complication rate among periods (all periods: 6 incidences, $15.8 \%, p=1.000$ ). Risk factors for longer WIT were screened per period by dividing the groups by the median WIT (17.2 min; Supplementary Table 1). Logistic regression analysis confirmed that male patients (odds ratio [OR], $6.182 ; 95 \%$ confidence interval [Cl], 1.101-34.700; $p=0.038)$ and a RENAL N-component score of $3(\mathrm{OR}, 6.500 ; 95 \% \mathrm{Cl}, 1.127-$ 34.484; $p=0.036$ ) were significantly associated with longer WIT in period 1 (Table 3 ). In period 2 , tumors larger than $33 \mathrm{~mm}(\mathrm{OR}, 6.500 ; 95 \% \mathrm{Cl}, 1.537-27.486 ; \mathrm{p}=0.011)$, a RENAL N-component score of 3 (OR, $5.000 ; 95 \% \mathrm{Cl}, 1.096-22.820 ; \mathrm{p}=0.038)$, and a total RENAL score of 8 or more (OR, $4.667 ; 95 \% \mathrm{Cl}$, 1.187-18.352; $p=0.027$ ) were significantly associated with longer WIT (Table 3 ). In period 3, a RENAL Lcomponent score of 3 was significantly associated with longer WIT (OR, 3.900; $95 \% \mathrm{Cl}, 1.004-15.276 ; \mathrm{p}$ $=0.044$; Table 3), but the tumor diameter and the total RENAL score were not (Supplementary Table 1). 
Table 1

Patient characteristics

\begin{tabular}{|c|c|c|c|c|c|c|}
\hline & & All cases & $\begin{array}{l}\text { All cases } \\
\text { by TH }\end{array}$ & 1st period & 2nd period & 3rd period \\
\hline & & $N=174$ & $N=114$ & 38 & 38 & 38 \\
\hline Age (year) & & $\begin{array}{l}64(33- \\
83)\end{array}$ & $\begin{array}{l}64(35- \\
83)\end{array}$ & $\begin{array}{l}62.4(37- \\
81)\end{array}$ & $\begin{array}{l}65.5(35- \\
82)\end{array}$ & $\begin{array}{l}64(39- \\
83)\end{array}$ \\
\hline \multirow[t]{2}{*}{ Sex (\%) } & Male & $129(74.1)$ & $85(74.6)$ & $28(73.7)$ & 32 (84.2) & $25(65.8)$ \\
\hline & Female & $45(25.9)$ & $29(25.4)$ & $10(26.3)$ & $6(15.8)$ & $13(34.2)$ \\
\hline BMI (kg/m2) & & $\begin{array}{l}24.5 \\
(18.7- \\
43.6)\end{array}$ & $\begin{array}{l}24.4 \\
(18.7- \\
38.3)\end{array}$ & $\begin{array}{l}24.2 \\
(19.2- \\
29.2)\end{array}$ & $\begin{array}{l}24.6 \\
(18.7- \\
38.3)\end{array}$ & $\begin{array}{l}24.6 \\
(18.9- \\
37.3)\end{array}$ \\
\hline \multirow[t]{2}{*}{ Laterality (\%) } & Right & 107 (61.5) & $69(60.5)$ & $26(68.4)$ & $26(68.4)$ & $17(44.7)$ \\
\hline & Left & $67(38.5)$ & 45 (39.5) & $12(31.6)$ & $12(31.6)$ & $21(55.3)$ \\
\hline Diameter (mm) & & $\begin{array}{l}29(11- \\
68)\end{array}$ & $\begin{array}{l}32(13- \\
68)\end{array}$ & $\begin{array}{l}26(13- \\
38)\end{array}$ & $\begin{array}{l}33.5(15- \\
60)\end{array}$ & $\begin{array}{l}39.5(15- \\
68)\end{array}$ \\
\hline $\begin{array}{l}\text { RENAL } \\
\text { nephrometry score }\end{array}$ & & $7(4-10)$ & $7(4-10)$ & $5(4-10)$ & $7(4-10)$ & $8(4-10)$ \\
\hline $\begin{array}{l}\text { Pre operative eGFR } \\
(\mathrm{mL} / \mathrm{min})\end{array}$ & & $\begin{array}{l}67.6 \\
(28.6- \\
164.7)\end{array}$ & $\begin{array}{l}69.4 \\
(28.7- \\
164.7)\end{array}$ & $\begin{array}{l}75.2 \\
(50.5- \\
143.5)\end{array}$ & $\begin{array}{l}64.7 \\
(28.7- \\
101.1)\end{array}$ & $\begin{array}{l}68.2 \\
(30.5- \\
164.7)\end{array}$ \\
\hline \multirow[t]{2}{*}{ Clinical T (\%) } & $1 \mathrm{a}$ & 147 (84.5) & 87 (76.3) & $37(97.4)$ & $30(78.9)$ & $20(52.6)$ \\
\hline & $1 b$ & $27(15.5)$ & 27 (23.7) & $1(2.6)$ & $8(21.1)$ & $18(47.4)$ \\
\hline
\end{tabular}


Table 2

Surgical outcomes of robot assisted partial nephrectomy

\begin{tabular}{|c|c|c|c|c|c|c|}
\hline & & $\begin{array}{l}\text { All } \\
\text { cases }\end{array}$ & $\begin{array}{l}\text { All } \\
\text { cases } \\
\text { by HT }\end{array}$ & $\begin{array}{l}1 \text { st } \\
\text { period }\end{array}$ & $\begin{array}{l}\text { 2nd } \\
\text { period }\end{array}$ & $\begin{array}{l}\text { 3rd } \\
\text { period }\end{array}$ \\
\hline median (range) & & $N=174$ & $N=114$ & $N=38$ & $N=38$ & $N=38$ \\
\hline \multirow[t]{2}{*}{ Approach (\%) } & Transperitoneal & $\begin{array}{l}114 \\
(65.5)\end{array}$ & $\begin{array}{l}69 \\
(60.5)\end{array}$ & $\begin{array}{l}31 \\
(81.6)\end{array}$ & $\begin{array}{l}27 \\
(71.1)\end{array}$ & $\begin{array}{l}11 \\
(28.9)\end{array}$ \\
\hline & Retroperitoneal & $\begin{array}{l}60 \\
(34.5)\end{array}$ & $\begin{array}{l}45 \\
(39.5)\end{array}$ & $7(18.4)$ & $\begin{array}{l}11 \\
(28.9)\end{array}$ & $\begin{array}{l}27 \\
(71.1)\end{array}$ \\
\hline Operative time (min) & & $\begin{array}{l}221 \\
(140- \\
399)\end{array}$ & $\begin{array}{l}223 \\
(140- \\
353)\end{array}$ & $\begin{array}{l}197.5 \\
(140- \\
353)\end{array}$ & $\begin{array}{l}231 \\
(153- \\
290)\end{array}$ & $\begin{array}{l}239.5 \\
(174- \\
328)\end{array}$ \\
\hline Operator & & 6 & 1 & 1 & 1 & 1 \\
\hline \multirow[t]{3}{*}{ Console time (min) } & All cases & $\begin{array}{l}143 \\
(42- \\
277)\end{array}$ & $\begin{array}{l}138.5 \\
(42- \\
277)\end{array}$ & $\begin{array}{l}140.5 \\
(64- \\
203)\end{array}$ & $\begin{array}{l}142 \\
(74- \\
200)\end{array}$ & $\begin{array}{l}136.5 \\
(42- \\
277)\end{array}$ \\
\hline & Transperitoneal & $\begin{array}{l}157 \\
(42- \\
262)\end{array}$ & & & & \\
\hline & Retroperitoneal & $\begin{array}{l}113 \\
(58- \\
277)\end{array}$ & & & & \\
\hline $\begin{array}{l}\text { Estimated blood loss } \\
(\mathrm{mL})\end{array}$ & & $\begin{array}{l}42(0- \\
1805)\end{array}$ & $\begin{array}{l}60(0- \\
1805)\end{array}$ & $\begin{array}{l}63(0- \\
1805)\end{array}$ & $\begin{array}{l}50.5(0- \\
1184)\end{array}$ & $\begin{array}{l}74.5(0- \\
736)\end{array}$ \\
\hline $\begin{array}{l}\text { Warm ischemia time } \\
\text { (min) }\end{array}$ & & $\begin{array}{l}17.3 \\
(9.2- \\
56)\end{array}$ & $\begin{array}{l}17.2 \\
(9.2- \\
56.0)\end{array}$ & $\begin{array}{l}17.4 \\
(9.3- \\
34.0)\end{array}$ & $\begin{array}{l}16.9 \\
(10.1- \\
56.0)\end{array}$ & $\begin{array}{l}17.3 \\
(10.9- \\
29.2)\end{array}$ \\
\hline $\begin{array}{l}\text { Intra-operative } \\
\text { complication (\%) }\end{array}$ & Satava $\geq 2$ & $3(1.7)$ & $1(0.9)$ & $1(2.6)$ & $0(0)$ & $0(0)$ \\
\hline $\begin{array}{l}\text { Postoperative } \\
\text { complication (\%) }\end{array}$ & $\begin{array}{l}\text { Clavien-Dindo } \\
\geq 2\end{array}$ & $\begin{array}{l}27 \\
(15.5)\end{array}$ & $\begin{array}{l}17 \\
(14.9)\end{array}$ & $5(13.2)$ & $6(15.8)$ & $6(15.8)$ \\
\hline $\begin{array}{l}\text { Positive surgical margin } \\
(\%)\end{array}$ & & $1(0.6)$ & $1(0.9)$ & $1(2.6)$ & $0(0)$ & $0(0)$ \\
\hline $\begin{array}{l}\text { eGFR at one months } \\
\text { after operation (\%) }\end{array}$ & $\geq 90 \%$ & $\begin{array}{l}121 \\
(69.5)\end{array}$ & $\begin{array}{l}73 \\
(64.0)\end{array}$ & $\begin{array}{l}29 \\
(76.3)\end{array}$ & $\begin{array}{l}28 \\
(73.7)\end{array}$ & $\begin{array}{l}16 \\
(42.1)\end{array}$ \\
\hline $\begin{array}{l}\text { Trifecta } \\
\text { accomplishment (\%) }\end{array}$ & & $\begin{array}{l}151 \\
(86.8)\end{array}$ & $\begin{array}{l}100 \\
(87.7)\end{array}$ & $\begin{array}{l}33 \\
(86.8)\end{array}$ & $\begin{array}{l}32 \\
(84.2)\end{array}$ & $\begin{array}{l}35 \\
(92.1)\end{array}$ \\
\hline Histology (\%) & Clear & $\begin{array}{l}128 \\
(73.6)\end{array}$ & $\begin{array}{l}84 \\
(73.7)\end{array}$ & $\begin{array}{l}27 \\
(71.1)\end{array}$ & $\begin{array}{l}33 \\
(86.8)\end{array}$ & $\begin{array}{l}24 \\
(63.2)\end{array}$ \\
\hline
\end{tabular}




\begin{tabular}{|c|c|c|c|c|c|c|}
\hline & & $\begin{array}{l}\text { All } \\
\text { cases }\end{array}$ & $\begin{array}{l}\text { All } \\
\text { cases } \\
\text { by HT }\end{array}$ & $\begin{array}{l}\text { 1st } \\
\text { period }\end{array}$ & $\begin{array}{l}\text { 2nd } \\
\text { period }\end{array}$ & $\begin{array}{l}\text { 3rd } \\
\text { period }\end{array}$ \\
\hline & Papillary & $\begin{array}{l}22 \\
(12.6)\end{array}$ & $\begin{array}{l}14 \\
(12.3)\end{array}$ & $4(10.5)$ & $3(7.9)$ & $7(18.4)$ \\
\hline & Chromophobe & $9(5.2)$ & $7(6.1)$ & $2(5.3)$ & $2(5.3)$ & $3(7.9)$ \\
\hline & AML & $7(4.0)$ & $5(4.4)$ & $3(7.9)$ & $0(0)$ & $2(5.3)$ \\
\hline & Oncocytoma & $3(1.7)$ & $3(2.6)$ & $2(5.3)$ & $0(0)$ & $1(2.6)$ \\
\hline & Xp11.2 & $1(0.6)$ & $1(0.9)$ & $0(0)$ & $0(0)$ & $1(2.6)$ \\
\hline & MTSCC & $1(0.6)$ & $0(0)$ & $0(0)$ & $0(0)$ & $0(0)$ \\
\hline Pathological T (\%) & $1 \mathrm{a}$ & $\begin{array}{l}145 \\
(83.3)\end{array}$ & $\begin{array}{l}90 \\
(78.9)\end{array}$ & $\begin{array}{l}31 \\
(81.6)\end{array}$ & $\begin{array}{l}33 \\
(86.8)\end{array}$ & $\begin{array}{l}26 \\
(68.4)\end{array}$ \\
\hline & $1 b$ & $\begin{array}{l}15 \\
(8.6)\end{array}$ & $\begin{array}{l}14 \\
(12.3)\end{array}$ & $1(2.6)$ & $5(13.2)$ & $8(21.1)$ \\
\hline & $3 a$ & $2(1.1)$ & $2(1.8)$ & $1(2.6)$ & $0(0)$ & $1(2.6)$ \\
\hline $\begin{array}{l}\text { HT, high-volume sur } \\
\text { angiomyolipoma; } \\
\text { and whether it has }\end{array}$ & $\begin{array}{l}\text { our hospital; ec } \\
\text { mucinous tubul } \\
\text { nearby tissue }\end{array}$ & $\begin{array}{l}\text { estima } \\
\text { nd spin }\end{array}$ & $\begin{array}{l}\text { glomer } \\
\text { cell car }\end{array}$ & $\begin{array}{l}\text { filtratio } \\
\text { ma; T, s }\end{array}$ & $\begin{array}{l}e ; A M L, \\
\text { f the pri }\end{array}$ & y tumor \\
\hline
\end{tabular}

Table 3

Risk factors longer WIT in the each period

\begin{tabular}{|c|c|c|c|c|c|c|}
\hline & & Risk category & OR & $95 \% \mathrm{Cl}$ & & $\mathrm{P}$ \\
\hline & & & & lower limit & upper limit & \\
\hline \multirow[t]{2}{*}{1 st period } & Gender & Male & 6.182 & 1.101 & 34.700 & 0.038 \\
\hline & $\mathrm{N}$ & 3 & 6.500 & 1.127 & 34.484 & 0.036 \\
\hline \multirow[t]{3}{*}{ 2nd period } & Tumor diameter (mm) & $33 \leq$ & 6.500 & 1.537 & 27.486 & 0.011 \\
\hline & $\mathrm{N}$ & 3 & 5.000 & 1.096 & 22.820 & 0.038 \\
\hline & Total score & $8 \leq$ & 4.667 & 1.187 & 18.352 & 0.027 \\
\hline \multirow[t]{2}{*}{ 3rd period } & L & 3 & 3.900 & 1.004 & 15.276 & 0.044 \\
\hline & Total score & $9 \leq$ & 2.444 & 0.654 & 9.130 & 0.184 \\
\hline
\end{tabular}


As the surgeon gained RALPN experience, the procedure was able to be used in more complex cases, such as patients with larger tumors and worse kidney function. However, the complication rate did not change throughout the study period. In the introductory period, male sex and a high RENAL N-component score were associated with longer WIT. In the intermediate period, a large tumor and high N-component and total RENAL scores were associated with longer WIT, but in the late period, only a high RENAL Lcomponent score was associated.

Over the past decade, robotics rapidly spread across all surgical fields, with tremendous innovation in urological surgeries [17]. Presently, robot techniques are routinely applied safely and effectively to urological operations. The success of RALPN may be primarily influenced by a surgeon's experience and confidence. In this study, the indication for RALPN for RCC was influenced by tumor size during the early period [18]. However, as the surgeon's experience with RALPN increased, the indication for RALPN expanded, eventually becoming the standard procedure for T1a and T1b RCC at our institution. Other studies have also reported similar data $[19,20]$. In our early period, tumors $<4 \mathrm{~cm}$ in diameter were suitable for the robotic platform, but tumors $>4 \mathrm{~cm}$ in size were also treated with RALPN in the later periods. In period 3, 47 \% of tumors were stage cT1b (18/38; Table 1), and RALPN was performed by our institute's most experienced surgeon. Furthermore, during the late period, patients with less kidney function, such as RCC in the solitary kidney, were also candidates for RALPN.

Objectively evaluating the difficulty of a partial nephrectomy has been attempted ever since the introduction of the laparoscopic partial nephrectomy. The RENAL score is a well-validated method for this purpose and is used to evaluate RALPN patients. However, these scores are primarily validated from laparoscopic partial nephrectomies and may be inadequate for assessing robotic procedure difficulties $[10,21]$. Nevertheless, each RENAL score component focused on representative points regarding the kidney tumor dissection. The $\mathrm{N}$-component indicates the nearness of the tumor to the collecting system or sinus. For a less experienced surgeon, an open collecting system and a large vessel injury would be troublesome [22], potentially resulting in longer WIT during the early experience period. The L-component indicates the tumor location relative to the polar lines. A high L-component score included hilar tumors in patients with a large tumor. In our late period, most cases were considered highly complex tumors because of their large diameter (median, $39.5 \mathrm{~mm}$ ) and high total RENAL score (median, 8). In these complicated cases, a central tumor lesion affected WIT because a major parenchymal defect across a central kidney lesion is difficult to cope with. To suture between surgical margins, a deep suture is needed to avoid parenchymal fracture and postoperative hemorrhage. However, this suture potentially causes injury to major vessels and the collecting system [23]. Thus, careful suturing is required, resulting in longer WIT [24].

Dissection and suturing skills evolve with increasing experience, and obtaining skills other than laparoscopic partial nephrectomy is a relatively rapid process. Zeuschner et al. reviewed RALPN outcomes and suggested that 35 cases were the minimum required number to acquire adequate RALPN skills [25]. The complication rate did not change throughout our study, which could be explained by the fact that the cases were carefully selected to match the surgeon's skill during each period. 
A pseudoaneurysm was the main complication in this study. As RALPN use increased, fewer pseudoaneurysm cases after laparoscopic partial nephrectomy were expected [26]. However, pseudoaneurysms remain at a low but constant rate in the robotic era [27]. In this study, all pseudoaneurysms cases were successfully managed by arterial embolization. Based on these findings derived and our study's complication rate, we conclude that RALPN is a safe procedure.

Our study had several limitations. First, this was a retrospective review of data from patients treated at a single institution, and multi-center, prospective studies are still needed. Second, Second, WIT risk evaluation was only investigated by RENAL score-related factors and clinical factors and did not include C-index or PADUA score. Third, long-term follow-up of kidney function was lacking. Almost all of our WIT was less than $25 \mathrm{~min}$, and it is uncertain if such a short WIT affects kidney function. Although this is not the first study on this topic, to our knowledge, ours is the first to assess WIT risk factors in complex patients. Furthermore, a single surgeon performed surgery on a sufficient number of patients, emphasizing the relevance of our study results.

\section{Conclusions}

RALPN is an effective and safe treatment that combines the advantages of a robotic platform with the standard oncological surgical strategies. A large tumor in the central lesion of a kidney may result in longer WIT, even when the procedure is performed by an experienced surgeon.

\section{Declarations}

\section{AUTHOR CONTRIBUTIONS}

Kazuyuki Numakura: Conceptualization, Methodology, Software, Statistical analysis, Writing- Reviewing and Editing;

Mizuki Kobayashi: Conceptualization, Data analysis, Reviewing and Editing;

Atsushi Koizumi: Data acquisition, Visualization, Investigation, Reviewing and Editing;

Soki Kashima: Data acquisition, Statistical analysis;

Ryohei Yamamoto: Data acquisition, Statistical analysis;

Taketoshi Nara: Data acquisition, Statistical analysis;

Mitsuru Saito: Data acquisition, Statistical analysis;

Shintaro Narita: Data acquisition, Statistical analysis;

Takamitsu Inoue: Data acquisition, Statistical analysis; 
Tomonori Habuchi: Conceptualization, Supervision, Manuscript editing.

\section{FUNDING}

This study was supported by Grants-in-Aid for Scientific Research, Japan (Grant No.: 17K11121 and 20K09553).

\section{ACKNOWLEDGMENTS}

We would like to thank Editage (www.editage.com) for English language editing.

\section{AUTHOR DISCLOSURE CONFLICT OF INTEREST}

Drs. Kazuyuki Numakura, Mizuki Kobayashi, Atsushi Koizumi, Soki Kashima, Ryohei Yamamoto, Taketoshi Nara, Mitsuru Saito, Shintaro Narita, Takamitsu Inoue, and Tomonori Habuchi have no conflicts of interest or financial ties to disclose.

\section{References}

1. Ljungberg B, Bensalah K, Canfield S, Dabestani S, Hofmann F, Hora M, Kuczyk MA, Lam T, Marconi L, Merseburger AS, Mulders P, Powles T, Staehler M, Volpe A, Bex A (2015) EAU guidelines on renal cell carcinoma: 2014 update. Eur Urol 67:913-924

2. Andrade HS, Zargar H, Caputo PA, Akca O, Kara O, Ramirez D, Haber GP, Stein RJ, Kaouk JH (2016) Five-year Oncologic Outcomes After Transperitoneal Robotic Partial Nephrectomy for Renal Cell Carcinoma. Eur Urol 69:1149-1154

3. Deng W, Li J, Liu X, Chen L, Liu W, Zhou X, Zhu J, Fu B, Wang G (2020) Robot-assisted versus laparoscopic partial nephrectomy for anatomically complex T1b renal tumors with a RENAL nephrometry score >/=7: A propensity score-based analysis. Cancer Med 9:586-594

4. Mikhail D, Sarcona J, Mekhail M, Richstone L (2020) Urologic Robotic Surgery. Surg Clin North Am 100:361-378

5. Hung AJ, Cai J, Simmons MN, Gill IS (2013) "Trifecta" in partial nephrectomy. J Urol 189:36-42

6. Volpe A, Blute ML, Ficarra V, Gill IS, Kutikov A, Porpiglia F, Rogers C, Touijer KA, Van Poppel H, Thompson RH (2015) Renal Ischemia and Function After Partial Nephrectomy: A Collaborative Review of the Literature. Eur Urol 68:61-74

7. Chronic Kidney Disease Prognosis C, Matsushita K, van der Velde M, Astor BC, Woodward M, Levey AS, de Jong PE, Coresh J, Gansevoort RT (2010) Association of estimated glomerular filtration rate and albuminuria with all-cause and cardiovascular mortality in general population cohorts: a collaborative meta-analysis. Lancet 375:2073-2081

8. Veccia A, Antonelli A, Uzzo RG, Novara G, Kutikov A, Ficarra V, Simeone C, Mirone V, Hampton LJ, Derweesh I, Porpiglia F, Autorino R (2020) Predictive Value of Nephrometry Scores in Nephronsparing Surgery: A Systematic Review and Meta-analysis. Eur Urol Focus 6:490-504 
9. Yao Y, Xu Y, Gu L, Liu K, Li P, Xuan Y, Gao Y, Zhang X (2020) The Mayo Adhesive Probability Score Predicts Longer Dissection Time During Laparoscopic Partial Nephrectomy. J Endourol 34:594-599

10. Kumar RM, Lavallee LT, Desantis D, Cnossen S, Mallick R, Cagiannos I, Morash C, Breau RH (2017) Are renal tumour scoring systems better than clinical judgement at predicting partial nephrectomy complexity? Can Urol Assoc J 11:199-203

11. Okhunov Z, Rais-Bahrami S, George AK, Waingankar N, Duty B, Montag S, Rosen L, Sunday S, Vira MA, Kavoussi LR (2011) The comparison of three renal tumor scoring systems: C-Index, P.A.D.U.A., and R.E.N.A.L. nephrometry scores. J Endourol 25:1921-1924

12. Hu JC, Treat E, Filson CP, McLaren I, Xiong S, Stepanian S, Hafez KS, Weizer AZ, Porter J (2014) Technique and outcomes of robot-assisted retroperitoneoscopic partial nephrectomy: a multicenter study. Eur Urol 66:542-549

13. Imbeault A, Pouliot F, Finley DS, Shuch B, Dujardin T (2012) Prospective study comparing two techniques of renal clamping in laparoscopic partial nephrectomy: impact on perioperative parameters. J Endourol 26:509-514

14. Omidele OO, Davoudzadeh N, Palese M (2018) Trifecta Outcomes to Assess Learning Curve of Robotic Partial Nephrectomy. JSLS 22

15. Tepeler A, Resorlu B, Sahin T, Sarikaya S, Bayindir M, Oguz U, Armagan A, Unsal A (2014) Categorization of intraoperative ureteroscopy complications using modified Satava classification system. World J Urol 32:131-136

16. Clavien PA, Barkun J, de Oliveira ML, Vauthey JN, Dindo D, Schulick RD, de Santibanes E, Pekolj J, Slankamenac K, Bassi C, Graf R, Vonlanthen R, Padbury R, Cameron JL, Makuuchi M (2009) The Clavien-Dindo classification of surgical complications: five-year experience. Ann Surg 250:187-196

17. Honda M, Morizane S, Hikita K, Takenaka A (2017) Current status of robotic surgery in urology. Asian J Endosc Surg 10:372-381

18. Motoyama D, Matsushita Y, Watanabe H, Tamura K, Suzuki T, Ito T, Sugiyama T, Otsuka A, Miyake H (2020) Initial learning curve for robot-assisted partial nephrectomy performed by a single experienced robotic surgeon. Asian J Endosc Surg 13:59-64

19. Vartolomei MD, Matei DV, Renne G, Tringali VM, Crisan N, Musi G, Mistretta FA, Russo A, Cozzi G, Cordima G, Luzzago S, Cioffi A, Di Trapani E, Catellani M, Delor M, Bottero D, Imbimbo C, Mirone V, Ferro M, de Cobelli O (2019) Robot-assisted Partial Nephrectomy: 5-yr Oncological Outcomes at a Single European Tertiary Cancer Center. Eur Urol Focus 5:636-641

20. Tufek I, Mourmouris P, Doganca T, Obek C, Argun OB, Tuna MB, Keskin MS, Kural AR (2017) RobotAssisted Partial Nephrectomy for T1b Tumors: Strict Trifecta Outcomes. JSLS 21

21. Rai BP, Patel A, Abroaf A, Suleyman N, Gowriemohan S, Prasad V, Vasdev N, Adshead J (2017) External validation of four nephrometry scores for trans-peritoneal robotic partial nephrectomy. Cent European J Urol 70:232-237

22. Mayer WA, Godoy G, Choi JM, Goh AC, Bian SX, Link RE (2012) Higher RENAL Nephrometry Score is predictive of longer warm ischemia time and collecting system entry during laparoscopic and 
robotic-assisted partial nephrectomy. Urology 79:1052-1056

23. Chavali JSS, Nelson R, Maurice MJ, Kara O, Mouracade P, Dagenais J, Reese J, Bayona P, Haber GP, Stein RJ (2018) Hilar Parenchymal Oversew: a novel technique for robotic partial nephrectomy hilar tumor renorrhaphy. Int Braz J Urol 44:199

24. Eyraud R, Long JA, Snow-Lisy D, Autorino R, Hillyer S, Klink J, Rizkala E, Stein RJ, Kaouk JH, Haber GP (2013) Robot-assisted partial nephrectomy for hilar tumors: perioperative outcomes. Urology 81:1246-1251

25. Zeuschner P, Meyer I, Siemer S, Stoeckle M, Wagenpfeil G, Wagenpfeil S, Saar M, Janssen M (2021) Three Different Learning Curves Have an Independent Impact on Perioperative Outcomes After Robotic Partial Nephrectomy: A Comparative Analysis. Ann Surg Oncol 28:1254-1261

26. Chung DY, Lee JS, Ahmad A, Chang KD, Ham WS, Han WK, Hong CH, Choi YD, Rha KH (2020) Lessons learned from clinical outcome and tumor features of patients underwent selective artery embolization due to postoperative bleeding following 2076 partial nephrectomies: propensity scoring matched study. World J Urol 38:1235-1242

27. Chavali JSS, Bertolo R, Kara O, Garisto J, Mouracade P, Nelson RJ, Dagenais J, Kaouk JH (2019) Renal Arterial Pseudoaneurysm After Partial Nephrectomy: Literature Review and Single-Center Analysis of Predictive Factors and Renal Functional Outcomes. J Laparoendosc Adv Surg Tech A 29:45-50

\section{Supplementary Files}

This is a list of supplementary files associated with this preprint. Click to download.

- SupplementTable1.docx 